\title{
La transformación de la tabla kantiana de las categorías en el Fundamento de toda la doctrina de la ciencia de J. G. Fichte de 1794/95
}

\author{
Transformation of the Kantian table of the \\ categories in Fichte's Foundations of the entire \\ science of knowledge of 1794/95
}

\author{
Emiliano AcostA \\ Vrije Universiteit Brussel/Ghent University
}

Recibido: 28-11-2014

Aceptado: 30-06-2015

\section{Resumen}

El presente artículo analiza la deducción fichteana de la categorías en el Fundamento de toda la doctrina de la ciencia de 1794/95 a la luz de la comprensión que tiene Fichte, durante los años de la génesis y publicación de esta obra, acerca de su relación con la filosofía kantiana con respecto a la cuestión de las categorías. Se argumenta que la deducción fichteana de las categorías en esta obra de 1794/95 transforma la tabla kantiana de las categorías en una tríada de tríadas (nueve categorías reunidas en los grupos de relación, cantidad, cualidad) en la que la determinación recíproca es la categoría fundamental, el grupo de la modalidad queda excluido y la distinción entre categorías matemáticas y dinámicas desaparece. El análisis de la deducción fichteana es seguido de una revisión crítica de las interpretaciones habituales sobre este tema.

Palabras clave: Kant, Fichte, (deducción de las) categorías, determinación recíproca.

\begin{abstract}
The present paper analyses Fichte's deduction of the categories in the Foundations of the entire Science of Knowledge (1794/95) from the perspective of
\end{abstract}


Fichte's view about the relation between his system and the Kantian philosophy concerning the question of the categories. It will be argued that Fichte modifies the Kantian table of the categories into a system of three categories groups, each of them composed by three categories. This implies, as it will be shown, the postulation of the reciprocal determination as first category and the exclusion from the categories system of both the modal categories and the distinction between dynamic and mathematic categories. The traditional interpretation of Fichte's deduction of the categories is discussed.

Keywords: Kant, Fichte, (deduction of the) categories, reciprocal determination.

\section{Introducción ${ }^{1}$}

El presente artículo ofrece una reconstrucción de la deducción fichteana de las categorías tal como se halla expuesta en el Fundamento de toda la doctrina de la ciencia de 1794/95 (de aquí en más GWL). ${ }^{2}$ Se analiza esta reconstrucción a la luz de la comprensión que hace Fichte de la relación entre su filosofía y la kantiana, en lo que respecta exclusivamente al tema de las categorías y durante los años de la génesis y publicación de la GWL. 3

\footnotetext{
${ }^{1}$ La obra de Fichte se cita según la edición de la Academia Bávara de las Ciencias: J. G. Fichte Gesamtausgabe der Bayerischen Akademie der Wissenschaften (de aquí en más GA), R. Lauth, H. Jacob et. al. (eds.), Stuttgart/Bad Cannstatt: Frommann-Holzboog, 1964 y ss.. En todos los casos las traducciones pertenecen al autor del presente artículo.

2 Fichte, J. G., Grundlage der gesammten Wissenschaftslehre als Handschrift für seine Zuhörer, 1794/95, GA I/2, 251-451. Traducción al español de este escrito: Fundamento de toda la doctrina de la ciencia (1794), trad., intr. y notas de Juan Cruz Cruz, Pamplona: 2005.

3 La exposición se restringe, pues, a la recepción de Fichte de la deducción kantiana de las categorías en la correspondencia, manuscritos de asistentes a sus lecciones y escritos (publicados en vida o no) de Fichte entre 1790 y 1795 , es decir, desde su primera lectura de las críticas kantianas hasta el momento de la publicación completa de su GWL. Escritos posteriores de Fichte son citados sólo con el objeto de contextualizar y demarcar el ámbito de validez dentro del desarrollo del pensamiento fichteano de aquello que el presente artículo expone. La delimitación propuesta se debe principalmente al hecho de que el intento de una reformulación de la deducción y de la tabla kantianas pierde, luego de 1795, su centralidad en el contexto del proyecto fichteano de una reelaboración de la filosofía trascendental. Esto es de verse en sus lecciones sobre la doctrina de la ciencia nova methodo (1796-1799), en las que Fichte propone un cambio en la exposición de su sistema que vuelve prescindible, como se explicará en las conclusiones (4), la deducción de las categorías tal como es presentada en la GWL. Se debe reconocer que estas lecciones brindan más y mejores elementos para lograr una comprensión amplia de la confrontación entre Fichte y Kant, principalmente por la madurez y autonomía conceptual que logra la doctrina de la ciencia en esos años. (Véase al respecto el número especial de Endoxa dedicado a la doctrina de la ciencia nova methodo, el cual consiste en un comentario parágrafo por parágrafo de los apuntes de Krause sobre las lecciones de 1798-1799, Fichte: la Doctrina de la ciencia nova methodo, Endoxa 30, 2012). La restricción del campo de estudio en el presente artículo busca exclusi-
} 
Se intentará mostrar en qué medida el proyecto de una reformulación de la deducción y sistematización kantianas de las categorías ocupa un lugar central en los primeros años del intento fichteano de reelaborar la filosofía de Kant. Se argumentará que la reformulación que ofrece Fichte en su GWL reordena las categorías en un sistema de tres tríadas: relación, cantidad y cualidad, excluyendo así el grupo de la modalidad de la tabla de las categorías. Esta nueva sistematización, se sostendrá, subordina todo el conjunto categorial bajo la categoría de determinación recíproca, cancela la distinción kantiana entre categorías matemáticas y dinámicas y coloca la deducción de las categorías, a diferencia de Kant, en un momento anterior a la deducción de las formas puras de la sensibilidad (espacio y tiempo) y de sujeto y objeto en sentido teórico. ${ }^{4}$ La propuesta de Fichte, como habrá de verse, implica además una modificación de la función de principio adjudicada por Kant a la apercepción pura y, en consecuencia, una redefinición de este concepto.

El presente trabajo se divide en cuatro secciones. En la primera sección se exponen los momentos principales en la recepción fichteana del sistema kantiano de las categorías entre 1790 y 1794 para contextualizar la deducción de las categorías de la GWL. La segunda sección de este artículo ofrece una reconstrucción y análisis del sistema fichteano de las categorías. La tercera sección analiza los problemas de la interpretación habitual de la tabla fichteana de las categorías. Por último, en la cuarta sección, se ofrecen las conclusiones de la presente investigación.

\section{El problema de la deducción de las categorías en la recepción fichteana del legado kantiano entre 1790 y 1794}

El motivo principal que anima el proyecto fichteano de una reelaboración de la filosofía kantiana proviene en primer lugar, como es sabido, de Reinhold. De él adopta Fichte tanto la idea de que debe buscarse aquel primer principio que Kant no pudo ofrecer como también el concepto general de sistema que indica la forma que debe poseer la filosofía transcendental para poder alcanzar el estatuto de ciencia. 5 Sin embargo, como también es sabido, Fichte está convencido de que aún después del aporte de Reinhold y, sobre todo, luego de las consideraciones críticas de

\footnotetext{
vamente identificar lo genuino de este momento del desarrollo del pensamiento fichteano con respecto al modo en que Fichte entiende su deducción de las categorías a la luz del legado kantiano.

4 Acerca de la diferencia entre sujeto y objeto en sentido teórico y práctico en la filosofía de Fichte véase Acosta, E., "Vier Bestimmungen des Nicht-Ich in der Jenenser Periode der Fichteschen Wissenschaftslehre", Rapic. S \& Pfeifer, M. (eds.) Das Selbst und sein Anderes: Festschrift für Klaus Erich Kaehler, München: Alber, 2009, pp. 98-108.

5 Véase entre otros comentarios Neuhouser, F., Fichte's Thoery of Subjectivity, Cambridge: Cambridge University Press, 1990, pp. 21 y ss. y Beiser, F., German Idealism. The Struggle against subjectivism: 1781-1801, Cambridge, Ma./London: Harvard University Press, 2002, pp. 226 y ss.
} 
Schulze y Maimon, la filosofía "aún no ha sido elevada al rango de ciencia evidente."6 Para lograr esto Fichte cree, al igual que Reinhold, que la filosofía debe hallar ese principio único desde el cual el sistema todo pueda ser deducido. ${ }^{7}$ Este motivo está operando también en la recepción crítica fichteana de la deducción kantiana de las categorías. Es interesante notar aquí que si bien Fichte ya en sus primeras lecturas de las tres críticas, en 1790, muestra ciertos reparos acerca de la claridad y orden de la exposición kantiana, ${ }^{8}$ sólo en 1793 , esto es, luego del encuentro con la filosofía de Reinhold, y con las críticas de Schulze y Maimon, llega a la conclusión de que el problema de la sistematicidad también está presente en la deducción kantiana de las categorías. ${ }^{9}$ En unas anotaciones acerca de la primera crítica kantiana, de noviembre o diciembre de 1793, Fichte escribe:

p. 104.5 [sc. KrV §10, B 104-105] surge la gran pregunta: ¿surge el concepto puro del entendimiento por medio de la síntesis (pura) o está dado para sustentar la síntesis?10

Ya a fines de 1793 Fichte entiende pues que la pregunta para comenzar una reelaboración de la deducción kantiana de las categorías debe indagar acerca del carácter genético de la deducción en relación con su principio. Esta pregunta se conecta con aquello que Fichte sostiene en otro manuscrito del mismo periodo, a saber, sus Meditaciones personales sobre filosofía elemental,11 escritas entre noviembre de

6 GA I/2, 109, Über den Begriff der Wissenschaftslehre oder der sogenannten Philosophie als Einladungsschrift zu seinen Vorlesungen über diese Wissenschaft, 1794. Existe traucción al español: Sobre el concepto de la doctrina de la ciencia, seguido de tres escritos sobre la misma disciplina, trad. B. Navarro, México: Instituto de Investigaciones Filosóficas, 2009.

7 GA I/2, 112, Über den Begriff der Wissenschaftslehre.

8 GA III/1, 168, 175 y 188-189, correspondencia con Fr. A. Weisshuhn de agosto o setiembre de 1790; del 27 setiembre de 1790, y, posiblemente, de noviembre de 1790 respectivamente.

9 De sus lecturas de las críticas kantianas en 1790 se conservan dos manuscritos, uno de los cuales es un resumen de algunos parágrafos de la doctrina transcendental de los elementos. (Der transzendentalen Elementalehre, zweiter Theil, 1790, GA II/1, 299-318) Al comentar tanto la tabla de los juicios como la de las categorías en este manuscrito Fichte no realiza observación alguna sobre el modo en que Kant deduce ambas tablas ni sobre el contenido y clasificación de los juicios y las categorías (GA II/1, 305-309). La definición kantiana de las categorías como "conceptos puros del entendimiento" (KrV B 105) tampoco es sometida a crítica (GA II/1, 308). Por último, el esquema de la tabla de las categorías que Kant presenta en el $\S 10$ de su primera crítica (B 106) es reproducido por Fichte sin modificación alguna (GA II/1, 308).

10 GA II/2, 328, Bei Lesung der K. KR. D. R. VFT. [sic], 1793.

11 Fichte, J. G., Eigne Meditationen über ElementarPhilosophie, 1793/94, GA II/3, 21-177. Acerca de este manuscrito pueden consultarse los siguientes estudios en español: Ivaldo, M., "La idea de filosofía trascendental en la primera doctrina de la ciencia", López Domínguez, V. (ed.) Fichte doscientos años después, Madrid: Editorial Complutense, 1996, pp. 11-24; Serrano Marín, V., "Reflexiones acerca del papel de las Eigne Meditationen para la formación del sistema trascendental de Fichte", Daimon, Revista de Filosofia, 9 (1994), pp. 39-49 y Metafisica y filosofia trascendental en el primer Fichte, Valencia: UPV, 2004. 
1793 y febrero de 1794. Allí Fichte se propone, entre otras tareas, “deducir las categorías y no aceptarlas como conceptos fundamentales", 12 lo cual en el contexto de este pasaje significa: conceptos que se aceptan sin demonstración alguna. La crítica a una tal aceptación de las categorías refiere aquí a Kant, dado que Fichte confiesa, a continuación del pasaje citado, no poder entender "cómo Kant pudo estar satisfecho con esto [sc. su deducción]."13

En este sentido, las Meditaciones personales atestiguan el comienzo de la reflexión fichteana sobre el concepto mismo de categoría, sobre la relación entre categorías y apercepción pura y sobre el carácter sistemático que una deducción de las categorías debe tener. ${ }^{14}$ Según Fichte se debe demostrar la necesidad de lo que él llama, siguiendo a Reinhold, los elementos de la filosofía trascendental, entre los cuales se hallan las categorías. ${ }^{15}$

Visto desde el problema de la sistematicidad de las categorías este escrito ofrece una sucesión de intentos de Fichte para lograr, mediante la demostración de la generación de las categorías a partir de la construcción de la relación de Yo y NoYo, una articulación sistemática entre las categorías y aquello que Fichte entiende como la síntesis originaria de la autoconsciencia. ${ }^{16}$ Los repetidos ensayos en este manuscrito para lograr una clara deducción y sistematización de las categorías ${ }^{17}$ así como también el número considerable de notas al margen de Fichte sobre esta cuestión, 18 muestran la importancia que este problema adquiere para Fichte en su intento de reelaborar la filosofía transcendental kantiana luego del ataque mortal del Enesidemo contra los sistemas de Kant y Reinhold.19

Sin ánimo de llevar a cabo un análisis exhaustivo de este manuscrito, de por sí complejo en su exposición y contenido, cabe señalar que, en lo que respecta al objeto de la presente investigación, en las Meditaciones personales se hallan ya motivos centrales, si bien sólo sugeridos, de la reelaboración fichteana del sistema categorial ofrecida en su GWL, tales como la identificación de realidad y negación con,

\footnotetext{
12 GA II/3, 41, Eigne Meditationen.

13 Ibídem.

14 GA II/3, 131, 134 y 146-147, Eigne Meditationen.

15 GA II/3, 26, Eigne Meditationen.

16 Véase entre otros pasajes GA II/3, 27, 40 y 50-51, Eigne Meditationen.

17 Véase entre otros pasajes GA II/3, 27 n., 38 n., 135, 142, 147 y 148, Eigne Meditationen.

18 Véase las notas al margen de Fichte en GA II/3, 27, 37, 38, 39, 41, 42, 44, 45, 49, 51, 54, 57, 58, 61, 69, 75, 112, 126, 128, 129, 147, 151 y 159, Eigne Meditationen.

19 GA III/2, 28, carta a H. Stephani de diciembre de 1793. Para una exposición de los primeros testimonios de Fichte sobre su lectura del Enesidemo véase la introducción de R. Lauth y H. Jacob a la Reseña del Enesidemo en GA I/2, 33-40, así como también Breazeale, D. "Fichte's 'Aenesidemus' Review and the Transformation of German Idealism”, The Review of Metaphysics, 34/3 (Marzo 1981), pp. 545-568.
} 
respectivamente, hacer (actividad) y padecer, ${ }^{20}$ y la primacía de la determinación recíproca dentro del sistema categorial.21

La cuestión de las categorías vuelve a aparecer en sus lecciones de Zúrich de febrero de 1794, esto es, meses antes de la publicación de la primera parte de la GWL (§§1-4) en junio del mismo año. Si bien estas lecciones son, como lo indica su título, "acerca del concepto de la doctrina de la ciencia", 22 Fichte realiza interesantes observaciones sobre la cuestión de las categorías. Por un lado, sugiere que cantidad y limitación surgen de la limitación recíproca de Yo y no-Yo, ${ }^{23}$ por otro, expone una primera reformulación de la tabla kantiana que lleva el título de "Ensayo de un orden mejorado de las categorías":

\section{Cantidad cualitativa - intensiva \\ Realidad \\ Negación \\ Limitación}

Categorias matemát[icas/]

Cantidad cuantitativa - extensiva
Unidad
Pluralidad
Totalidad

Cantidad cuantitativa - extensiva

Unidad

Totalidad

\section{Cualidad cuantitativa relativa \\ Substancia y accidente \\ Causa y efecto \\ Hacer y padecer}

Categorías dinámicas

Cualidad cuantitativa modal

Posibilidad - Imposibilidad

Existencia - Inexistencia

Necesidad - Contingencia ${ }^{24}$

Si bien el esquema cuádruple y la división entre categorías matemáticas y dinámicas de la tabla kantiana se conserva, lo cual, como se verá en (4), desaparece en la GWL, este ensayo de una nueva tabla de las categorías introduce un cambio significativo para el posterior desarrollo de esta cuestión en la GWL, a saber, la identificación de los grupos de la cualidad y la cantidad como modos en que se da la determinación cuantitativa.

En estos intentos de reelaborar la deducción kantiana de las categorías puede verse que Fichte ya posee una definición propia de categoría. Al inicio de sus

\footnotetext{
$20 \mathrm{GA} \mathrm{II} / 3,135$, Eigne Meditationen.

21 GA II/3, 38 n., Eigne Meditationen.

22 Fichte, J. G., Züricher Vorlesungen über den Begriff der Wissenschaftslehre, Nachschrift Lavater, 1794, GA IV/3, 19-41. Acerca de la génésis y estructura conceptual de este manuscríto véase: Radrizzani, I., "Fichtes 'erste' Wissenschaftslehre”, Fichte-Studien, 16 (1999), pp. 403-431.

23 GA IV/3, 31, Züricher Vorlesungen über den Begriff der Wissenschaftslehre.

24 GA IV/3, 48, Excerpt aus den Züricher Vorlesungen über Wissenschaftslehre, Abschrift Baggesen, 1794.
} 
Meditaciones personales Fichte las coloca dentro del grupo de los "modos de actuar y padecer de nuestro Yo". 25 Los modos en que actúa y padece el Yo son para Fichte a su vez acciones. De allí que defina en este manuscrito los conceptos puros del entendimiento como "procedimientos [Proceduren] realizados junto con el objeto de la intuición externa". ${ }^{26} \mathrm{El}$ carácter normativo de las categorías aparece en una nota sobre el grupo de la relación, en la que Fichte las define como "leyes" según las cuales "es pensado todo lo que puede ser sentido [alles empfindbare]".27 Fichte especifica el carácter activo de las categorías definiéndolas como acciones de relación: acciones por medio de las cuales Yo, No-Yo y consciencia se relacionan entre sí. ${ }^{28}$ Entender las categorías como acciones de relación permite a Fichte sugerir su vínculo genético con la unidad sintética de apercepción entendida como el relacionar del que surgen las acciones particulares de relación:

Contraponer e identificar a la vez significa relacionar. ¿Acaso la unidad sintética de la apercepción? ¿No consiste ella continuamente en relacionar?29

Esta nuevo significado de categoría se refleja en la GWL donde 'categoría' mienta una acción originaria particular del Yo, y por ende pre-consciente en cuanto anterior al surgimiento de consciencia y autoconsciencia empíricas. ${ }^{30}$ Categoría es según Fichte una acción que establece una ley para determinar todo contenido de consciencia. Cada categoría posee, entonces, un doble carácter: normativo y performativo a la vez. Ellas son los conceptos más generales que indican el modo en que debe darse todo contenido de consciencia y, a la vez, son acciones sintéticas originarias del Yo. Dicho con palabras de Fichte: ellas son "modos de acción"31 y "conceptos sintéticos". 32 Las categorías kantianas poseen, ciertamente, también sentido normativo, pero para Fichte, como sostendrá años más tarde, no son deducidas efectivamente como acciones originarias constitutivas de las leyes de la predicación del ser, sino obtenidas "por medio de abstracción de los objetos, luego: únicamente a partir de la experiencia." 33

25 GA II/3, 22, Eigne Meditationen.

26 GA II/3, 129, Eigne Meditationen.

27 GA II/3, 128, Eigne Meditationen.

28 De allí que en las Meditaciones Fichte llame a la parte teórica "doctrina de la esencia de las relaciones [Beziehungswesenlehre]". (GA II/3, 91)

29 GA II/3, 40, Eigne Meditationen.

30 Véase al respecto Metz, W., Kategoriendeduktion und produktive Einbildungskraft in der theoretischen Philosophie Kants und Fichtes, Stuttgart/Bad Cannstatt: Fromann Holzboog, 1991, p. 203; y Jürgensen, S., "Die Unterscheidung der Realität in Fichtes Wissenschaftslehre von 1794", FichteStudien 6 (1994), p. 51.

31 GWL, GA I/2, 282.

32 GWL, GA I/2, 299.

33 GA I/4, 201 Erste Einleitung in die Wissenschaftslehre, 1797. Si bien se trata de una afirmación proveniente de los años en que Fichte ya dictaba sus lecciones acerca de la doctrina de la ciencia nova 
Kant, según Fichte, toma las categorías "en el más bajo de sus niveles", donde "ya están aplicadas inmediatamente a los objetos". La doctrina de la ciencia, por el contrario, "deduce efectivamente a partir de las leyes fundamentales de la inteligencia el sistema de los modos necesarios de actuar y con él a la par las representaciones objetivas que surgen a través de esto, y así hace surgir paulatinamente ante los ojos del lector o del oyente todo el ámbito de nuestras representaciones." 34

La recepción crítica que hace Fichte de la deducción kantiana de las categorías se inicia, como se ha indicado, con la pregunta acerca del origen de las categorías a partir de un único principio. Como se ha mostrado, esta pregunta posee un lugar central en los primeros pasos que da Fichte en la elaboración de su sistema entre noviembre de 1793 y febrero de 1794. A partir de lo expuesto, Fichte parece tener especial interés no sólo en demostrar el vínculo genético entre las categorías y el principio del sistema todo, sino además en lograr un nuevo ordenamiento de la tabla kantiana de las categorías. Estos dos motivos guían, como se verá en la sección siguiente, la deducción de las categorías en la GWL.

\section{La deducción de las categorías en GWL}

\subsection{El inicio de la deducción}

La deducción, como habrá de verse, se inicia y desarrolla en su totalidad en el $\S 4$ de la GWL, en el que la génesis de las categorías es (re)construida a partir del teorema del saber teórico: "el Yo se pone como limitado por el No-Yo."35 Esto parece, sin embargo, no concordar con aquello que Fichte lleva a cabo en los $\S 1$ 1-3 de la GWL. Pues, en los $\S \S 1$ y 2 Fichte introduce las categorías de realidad y negación ${ }^{36}$ y al final del $\S 3$ reúne las categorías de cualidad y cantidad bajo "la categoría [sic] de la determinación [Bestimmung]".37 Como se expondrá en (5), la lectura habitual de la deducción fichteana de las categorías quiere ver en estas indicaciones de Fichte la primera parte de la deducción de las categorías, sin atender a las problemas que genera tal interpretación. En la primera parte de su GWL, Axiomas de toda la doctrina de la ciencia (§§1-3), Fichte, sin embargo, sólo enumera los elementos que luego, en la segunda parte, Fundamento del saber teórico (\$4), deduce genéticamente, lo cual en el contexto de la GWL significa: demostrar el modo en que ellas surgen inmediatamente de la acción sintética que las precede y mediata-

\footnotetext{
methodo, el contenido de esta afirmación y del pasaje que luego se cita en el cuerpo del texto no contradicen aquello que puede verse en la GWL acerca de este aspecto de las categorías fichteanas.

34 Ibídem.

35 GWL, GA I/2, 285.

36 GWL, GA I/2, 261 (realidad) y 267 (negación).

37 GWL, GA I/2, 282.
} 
mente, primero, de la unidad sintética expresada en el teorema del saber teórico y, en último término, de la actividad del Yo en cuanto sujeto absoluto. ${ }^{38}$ De allí que Fichte crea necesario aclarar que la primera síntesis del saber teórico en el §4, esto es, el primer paso en la deducción de las categorías, consiste en establecer "clara y distintamente" aquello que de algún modo sólo ha sido indicado en el §3.39

38 GA I/2, 262, 273-274 y 283. Véase también GA I/2, 114-115, Über den Begriff der Wissenschaftslehre. Ciertamente en estos años Fichte aún no ha desarrollado totalmente el método genético-constructivo que caracteriza su sistema a partir de las lecciones sobre la doctrina de la ciencia nova methodo. En la GWL el término 'genético' aparece por primera vez en el $\S 5$ (GA I/2, 404 y 432). Esto ha dado lugar a interpretaciones que creen ver aquí un cambio metodológico entre la primera $(\S \S 1-4,1794)$ y la segunda entrega $(\S \S 5-11,1795)$ de la GWL. (G. Meckenstock. "Beobachtungen zur Methodik in Fichtes Grundlage der gesammten Wissenschaftslehre", FichteStudien 10 (1997) p. 77; J. Beeler-Port "Zur Stellenwert der Grundlage aus der Sicht von 1804", Fichte-Studien 10 (1997), p. 339) Una lectura detenida de la obra en conjunto muestra que en realidad la introducción de este término no es sino una explicitación parcial de lo que Fichte ha estado llevando a cabo en los primeros parágrafos de su GWL. Por otra parte, debería atenderse al problema central de la versión, aún no acabada, del método genético en la GWL, a saber: la ausencia de la intuición intelectual en cuanto garante de la certeza y del contenido de la génesis que Fichte está reconstruyendo. Mientras en las Meditaciones personales, las lecciones de Zúrich y la Reseña del Enesidemo este concepto aparece en la explicación general de su método (GA II/3, 24, GA IV/3, 34 y GA I/2, 48, 57 y 65 , respectivamente), en la GWL (como también en Sobre el concepto de la doctrina de la ciencia) el mismo está ausente. Todo parece indicar, empero, que esto obedece sencillamente al hecho de que Fichte quiere evitar "una polémica abierta contra Kant y Reinhold". (Radrizzani, I., op. cit. p. 430) En sus lecciones sobre la doctrina de la ciencia nova methodo Fichte sostiene que este concepto sí se halla operando en la GWL, si bien el modo en el que se arriba a él es inverso al que propone en estas lecciones. (GA IV/3, 349 Fichtes Vorlesungen über die Wissenschaftslehre, gehalten zu Jena im Winter 1798/99, Nachschrift Krause, GA IV/2, 33, Wissenschatslehre nach den Vorlesungen von Hr. Pr. Fichte [ca. 1796-1799], Nachschrift Halle) De todos modos, esta afirmación de Fichte no debe soslayar el hecho de que el uso explícito de este concepto no sólo tiene consecuencias más que significativas en el desarrollo de su sistema (como es de verse tanto en las Meditaciones personales como en la doctrina de la ciencia nova methodo), sino que además evita la oscuridad de la exposición de la GWL. En la carta a Reinhold del 2 de julio de 1795 Fichte reconoce que esta falta de claridad en la GWL se debe a no haber introducido en este escrito la apelación a la intuición intelectual, esto es, a "la intuición interna del Yo a través de sí mismo" como contenido de la proposición "que es el alma de mi sistema: el Yo se pone a sí mismo sin más". (GA III/2, 344)

El uso del término 'deducción genética' en Fichte comienza a afianzarse en la Zweite Einleitung in die Wissenschaftslehre de 1797 (GA I/4, 248). Como se ha indicado más arriba, es en las lecciones sobre la doctrina de la ciencia nova methodo que Fichte muestra haber logrado un concepto definitivo de este método y lo emplea por primera vez de modo consecuente para la exposición de su doctrina de la ciencia. En su System der Sittenlehre la oposición deducción genética/deducción fáctica ya es parte esencial de la definición del método genético (GA I/5, 33). A partir de 1804 Fichte utiliza este término para designar el método por antonomasia de su filosofía (Vorlesungen der W.L. im Winter 1804, GA II/7, 69; Die Wissenschaftslehre [II. Vortrag im Jahre 1804], GA II/8, 43; y 3ter Cours der W.L. 1804, GA II/7, 306 y ss).

$39 \mathrm{GWL}, \mathrm{GA}$ I/2, 288. Esta manera de proceder puede hallarse en versiones posteriores de la doctrina de la ciencia. Ella corresponde con la distinción que Fichte años más tarde establece entre deducción fáctica y deducción genética (véase a modo de ejemplo GA II/11, 309, 315-316 y 320, Wissenschaftslehre 1810). 


\subsection{Consideraciones generales}

La deducción fichteana es, en sentido estricto, una reconstrucción, es decir, una exposición que busca reproducir la generación originaria del Yo que se supone ya acontecida. ${ }^{40}$ Esta reconstrucción se despliega en una serie de teoremas y conceptos, anunciada en el $\S 4$ y deducida a partir de la tríada axiomática establecida al inicio de la GWL (§§1-3). Dentro de esta estructura sistemática, desarrollada a partir del concepto de sistema expuesto en Sobre el concepto de la doctrina de la ciencia, ${ }^{41}$ el principio de la deducción, a saber, el teorema del saber teórico, ${ }^{42}$ se deduce del tercer y último axioma del sistema (\$3). Este teorema junto con el teorema principal del saber práctico ${ }^{43}$ son las dos proposiciones que componen el tercer axioma. Fichte afirma que en el tercer axioma se halla el "contenido para toda posible síntesis futura". ${ }^{44} \mathrm{El}$ tercer axioma, además de contener los teoremas del saber teórico y del saber práctico y ser, por consiguiente, el origen de las dos partes (teórica y práctica) de la GWL, representa la síntesis fundamental del sistema, la cual viene exigida por los dos primeros axiomas $(\S \S 1-2) .45$ En este sentido, el tercer axioma constituye el núcleo del sistema fichteano de 1794/95. Él es punto de culminación del establecimiento de los axiomas del sistema y punto de partida del despliegue de los saberes teórico y práctico. Así, el tercer axioma comunica la axiomática de la GWL con el desarrollo del sistema, tal como lo muestra la estructura básica quíntuple 46 de esta obra, compuesta por tres axiomas (Grundsätze) ${ }^{47}$ y dos teoremas (Lehrsätze):

40 GWL, GA I/2, 283-284. Véase también GA I/2, 126, 140 y 146-147, Über den Begriff der Wissenschaftslehre

41 GA I/2, 114-117 y 119-123, Über den Begriff der Wissenschaftslehre.

$42 \mathrm{Si}$ bien es cierto que las categorías, al igual que todos los elementos del sistema fichteano, son deducidas del "Yo en cuanto sujeto absoluto" (GA I/2, 262), este teorema describe la acción concreta del Yo de la que las categorías surgen y, en este sentido, cumple la función de ser el principio sensu stricto de la deducción de las categorías.

43 En la GWL el saber práctico se articula en siete teoremas (\$§5-11), a diferencia del saber teórico que consiste básicamente en el despliegue de un único teorema (\$4).

44 GWL, GA I/2, 283.

45 GWL, GA I/2, 268.

46 Si bien el término "quintuplicidad" (Fünffachheit) pertenece en sentido estricto al vocabulario de la filosofía fichteana luego de 1800, ya en el periodo de Jena, y en particular en la GWL, el pensamiento fichteano opera con el esquema conceptual de la quintuplicidad. Este tema ha sido tratado con detenimiento por A. Philonenko (La liberté humaine dans la philosophie de Fichte, Paris: Vrin, 1999, pp. 96 y ss.), si bien Philonenko no menciona la quintuplicidad que aquí se reconstruye. En este sentido, esta "síntesis quíntuple" entre axiomas y teoremas sirve de complementación al invalorable aporte de Philonenko para la comprensión de la estructura de la GWL.

En lo que se refiere a la estructura quíntuple del sistema fichteano de las ciencias filosóficas durante los años de Jena W. Janke y A. Philonenko proponen dos ordenamientos posibles. Según Janke el sistema se compone de una ciencia de las ciencias (doctrina de la ciencia) y cuatro ciencias filosóficas 


\begin{tabular}{|c|c|}
\hline $\begin{array}{c}\text { Primer axioma } \\
\text { El Yo se pone a sí mismo sin más }\end{array}$ & $\begin{array}{c}\text { Segundo axioma } \\
\text { Al Yo es contrapuesto sin más un No- } \\
\text { Yo }\end{array}$ \\
\hline \multicolumn{2}{|c|}{$\begin{array}{c}\text { Tercer axioma } \\
\text { El Yo contrapone en el Yo al Yo divisible un No-Yo divisible } \\
\text { (síntesis fundacional) }\end{array}$} \\
\hline $\begin{array}{c}\text { Teorema del saber teórico } \\
\text { El Yo se pone como limitado por el } \\
\text { No-Yo }\end{array}$ & $\begin{array}{c}\text { Teorema del saber práctico } \\
\text { El Yo pone el No-Yo como limitado } \\
\text { por el Yo }\end{array}$ \\
\hline
\end{tabular}

El orden en que se deducen los elementos de la doctrina de la ciencia muestra que Fichte, a diferencia de Kant, ubica la deducción de las categorías en un momento anterior a la deducción de las formas puras de la sensibilidad (espacio y tiempo) y a toda determinación del objeto. Como habrá de verse, Fichte entiende que en la deducción de las categorías se debe abstraer completamente de espacio y tiempo así como también de sujeto y objeto en sentido teórico.

Como se mostrará, Fichte deduce las categorías en cuanto síntesis que resuelven las contradicciones contenidas tanto en el teorema del saber teórico en general como en las proposiciones que lo componen en particular. Cada paso en la deducción consiste básicamente en dos operaciones: i) identificación de la antítesis contenida en el último miembro obtenido y ii) postulación de una síntesis particular como solución a la antítesis identificada. Cada nueva síntesis es, como habrá de verse, determinada como un nuevo eslabón en la cadena causal que da origen al Yo.

particulares: filosofía de la naturaleza, filosofía de la religión, doctrina de las costumbres y doctrina del derecho. (Historische Dialektik. Destruktion dialektischer Grundformen von Kant bis Marx, Berlin: De Gruyter, 1977 p. 102). Según Philonenko el sistema consiste en una complementación entre tres obras de Fichte y aquello que Fichte mismo no lleva a cabo y adopta, según Philonenko, de la primera y tercera críticas kantianas. De este modo el sistema se estructura en una quintuplicidad compuesta por la GWL en cuanto fundamentación del sistema, y cuatro ciencias subordinadas: filosofía teórica (Crítica de la razón pura), filosofía práctica (Sistema de la doctrina de las costumbres según principios de la doctrina de la ciencia, 1798), derecho natural (Fundamento del derecho natural según principios de la doctrina de la ciencia, 1796/97) y, por último, estética (primera parte de la Crítica del Juicio). (op. cit. p. 99)

47 Suele traducirse 'Grundsatz' al español como 'principio', 'primer principio' o 'proposición fundamental' (Grund-Satz). Sin embargo, este término significa, en el alemán académico del siglo XVIII, axioma. Esta acepción de 'Grundsatz' proviene de Wolff, quien usa este término para traducir el término latino 'axioma' y su correspondiente en griego. (Véase definición 'b' de 'Grundsatz' en el Deutsches Wörterbuch von J. Grimm und W. Grimm, http://woerterbuchnetz.de/ DWB/?sigle=DWB\&mode=Vernetzung\&lemid $=$ GG31228\#XGG31228, consultado el 2 de junio de 2015) Que Fichte en la GWL utiliza 'Grundsatz' para mentar 'axioma' es de verse en el hecho de que este término en la GWL refiere a las proposiciones que, como todo axioma, fundamentan el sistema, no son deducidas y de las cuales se deducen los 'Lehrsätze' (teoremas) del sistema. (W. Class \& A. K. Soller. Kommentar zu Fichtes Grundlage der gesamten Wissenschaftslehre, Fichte-StudienSupplementa 19, Amsterdam/New York: Rodopi, 2004, p. 23) 
Dos hechos, según Fichte evidentes de por sí, regulan esta dialéctica generativa 48 : la unidad efectiva de la (auto)consciencia y el carácter absoluto del Yo. ${ }^{49} \mathrm{La}$ primera es aquello que debe ser explicado. La explicación, por ende, no debe contradecir la unidad de la consciencia. El carácter absoluto del Yo sirve de postulado fundamental del sistema e idea reguladora del desarrollo todo del sistema. La espontaneidad del Yo y su carácter de "sujeto absoluto" 50 es aquello que exige que haya síntesis y que ejecuta cada una de ellas. Así, por ejemplo, en el caso de la síntesis originaria ( $(3)$ Fichte aclara que

si bien los conceptos contrapuestos [sc. Yo y No-Yo en sentido absoluto] están dados por medio de los dos primeros axiomas, la exigencia de que deban ser unificados está contenida en el primero [de los axiomas]. ${ }^{51}$

Por otra parte, la unificación acontecida es una acción del Yo que Fichte llama "acción Y, un limitar ambos contrapuestos mutuamente". ${ }^{52}$ En lo que concierne a

48 'Dialéctica generativa' no es, por cierto, un término perteneciente al vocabulario técnico de Fichte. El uso de este término en el presente artículo hace referencia al carácter dialéctico en el método fichteano de deducción en la GWL. El mismo consiste, en primer lugar, en la dinámica de tesis - antítesis - sintesis, con la que se inicia la GWL, y la posterior sucesión de la dinámica sintesis - antítesis sintesis, que caracteriza el desarrollo de la GWL. (GA I/2, 274-276) En segundo lugar, lo dialéctico aparece también en la GWL en el hecho de que en cada elemento ya se encuentra de algún modo operando su opuesto en cuanto condición para su determinación (por ejemplo GA I/2, 356 en la que Fichte muestra la interdependencia entre la actividad/espontaniedad del Yo y el choque-Anstoss-). Esta metodología de llevar cada elemento hasta su opuesto o hasta su contradicción se refleja también en el modo en que cada nueva antítesis o contradicción aparece, esto es: mediante una reflexión sobre la tesis (por ejemplo GA I/2, 269, §2. A. 5. donde Fichte muestra que una reflexión sobre el primer axioma tomado absolutamente lleva a su anulación) o sobre cada nueva síntesis (por ejemplo GA I/2, 283, donde Fichte sostiene que la reflexión sobre la síntesis originaria hará surgir en la deducción todas los atributos de los conceptos de Yo y No-Yo). Lo dialéctico de la deducción ya se halla presente en sus Meditaciones personales: "De acuerdo con el método esta tesis tendría que ser reconducida a una contradicción, la cual tendría que ser cancelada sólo por medio de un nuevo hecho sintético." (GA II/3, 135) Acerca del carácter dialéctico del método de la doctrina de la ciencia véase Janke, W., Historische Dialektik, pp. 100-209; "Limitative Dialektik. Überlegungen im Anschluss an die Methodenreflexion in Fichtes Grundlage 1794/95 §4 (GA I, 2, 283-285)", Fichte-Studien 9 (1990) pp. 9-24; Vom Bilde des Absoluten. Grundzüge der Phänomenologie Fichtes, Berlin: De Gruyter, 1993, pp. 187-212; Lauth, R., "Der Ursprung der Dialektik in Fichtes Philosophie", Holz, H. H., Lapin, N. I. \& Sandkühler, H. J. (eds.), Die Dialektik und die Wissenschaften - Philosophische Fragen moderner Entwicklungskonzeptionen, Köln: Pahl-Rugenstein, 1986, pp. 135-147; Seebohm, T. M., "Fichte's Discovery of Dialectic Method", Breazeale, D., \& Rockmore, T. (eds.) Fichte Historical Contexts/Contemporary Controversies, Atlantic Heigts, NJ: Humanity Books, 1994, pp. 17-42; y Limnatis, N., German Idealism and the Problem of Knowledge: Kant, Fichte, Schelling and Hegel, New York/Doodrecht: Springer, 2008, pp. 99-109.

49 GWL, GA I/2, 287.

$50 \mathrm{GWL}, \mathrm{GA} \mathrm{I} / 2,262$.

51 GWL, GA I/2, 270.

52 Ibídem. 
las síntesis restantes y, por ende, al sistema en general, Fichte vuelve a indicar la función regulativa determinante del Yo del primer axioma:

La necesidad de contraponer y unir de un modo determinado reside inmediatamente en el tercer axioma: la necesidad de unir en general, en el primero, sumo, incondicionado sin más. La forma del sistema se funda en la síntesis suprema, que deba haber un sistema en general, [se funda] en la tesis absoluta. 53

En lo que concierne al rol de ejecutor del Yo en cada una de las síntesis de la GWL baste con recordar que Fichte busca precisamente demostrar cómo lo activo del Yo está presente y se conserva en cada una de las síntesis.

La deducción fichteana de las categorías se articula en tres síntesis fundamentales, cada una de las cuales refiere a una categoría de relación: determinación recíproca, causalidad y sustancialidad. Mediante la primera síntesis son deducidos además los grupos de la cualidad y cantidad. Cada síntesis implica, a su vez, una nueva determinación de realidad y negación y la preeminencia de uno de los momentos de la cantidad. De este modo el sistema categorial se estructura como una tríada de tríadas de la que la modalidad no participa. 54

\subsection{Primera síntesis: determinación recíproca, realidad y negación relativas (magnitudes) y momento cuantitativo de la totalidad}

Fichte inicia la deducción de las categorías distinguiendo las dos proposiciones que componen el teorema del saber teórico e identificando la contradicción que se da entre ellas. Éstas son: "el Yo se pone como determinado por el No-Yo" y "el Yo se determina". 55 Mientras en la primera proposición lo determinante es el No-Yo, en la segunda, lo determinante es el Yo. Ambas proposiciones, siguiendo a Fichte, se contradicen. Ellas, sin embargo, conforman el teorema del saber teórico, el cual ha sido deducido del tercer axioma. Si éste es válido, sostiene Fichte, también deben serlo las proposiciones que lo componen. De no ser así, la GWL daría como resultado la anulación de la unidad de la (auto)consciencia. El teorema del saber teórico

\footnotetext{
53 GWL, GA I/2, 276. Véase además GWL, GA I/2, 276-278 y 284.

54 Como se verá más adelante (al final de 2.6) la exclusión de la modalidad no es arbitraria, sino que obedece al hecho de la redefinición fichteana de categoría en sentido estricto, a saber, en cuanto acción originaria de la razón en su autoconstitución, proceso que Fichte sitúa en una fase anterior a la deducción del objeto y de las formas puras de la sensibilidad. En este sentido, la modalidad no puede formar parte del sistema categorial fichteano, ya que, siguiendo el orden fichteano de deducción, actualidad, posibilidad y necesidad suponen, dentro del horizonte de sentido abierto por la GWL, tanto la posición en el Yo de un objeto (en sentido teórico) como la deducción de espacio y tiempo en cuanto formas puras de la sensibilidad. (GA I/2, 360 y s., 367, 370, 374 y 378)
}

55 GWL, GA I/2, 287. 
no debe anularse, si la unidad de la consciencia tampoco debe ser anulada: por consiguiente tenemos que intentar unificar los opuestos señalados. 56

Fichte presupone, entonces, que en el surgimiento real del Yo se han dado una serie de acciones originarias que han resuelto esta contradicción. La deducción debe, por lo tanto, reconstruir estas acciones. La acción originaria que resuelve esta contradicción es, como ha de verse, la síntesis de la determinación recíproca. Esta síntesis implica, como se expone más adelante, la deducción efectiva de los grupos de la cualidad y de la cantidad. Éstos, por cierto, ya habían sido obtenidos en los $\S \S$ 1-3, si bien sólo por medio de abstracción de las acciones originarias de poner, contraponer y limitar. 57

La contradicción entre las proposiciones que componen el teorema teórico consiste en que cada una predica del Yo o bien realidad (ser sujeto de una acción) o bien negación (ser objeto de una acción) en sentido absoluto, es decir, realidad o negación sin más. Mientras en la primera proposición se describe al Yo como objeto (pasividad) de una acción del No-Yo, en la segunda se afirma el carácter de sujeto (actividad) del Yo.

Ambas proposiciones se vuelven compatibles cuando realidad y negación son concebidas en sentido relativo. Anulando el carácter excluyente (el ser "sin más") de las mismas se posibilita que ambas entren en relación mutua, de modo que pueda predicarse a la vez realidad y negación del Yo. Para esto debe ingresarse un tercer concepto que subsuma realidad y negación en cuanto especificaciones del mismo y suprima así la diferencia absoluta entre ambas. Este concepto es, según Fichte, la categoría de cantidad. 58

Fichte entiende la cantidad de dos modos: como la acción de cuantificar la cualidad y como el resultado de esta acción, es decir, como la totalidad de los contenidos posibles de consciencia. La cantidad refiere así al acto generativo de todo contenido posible de consciencia en general y a este contenido mismo. Ambos modos de considerar la cantidad refieren, sin embargo, a una misma función en la deducción: subsumir realidad y negación bajo un mismo género.

La acción de cuantificar la cualidad transforma realidad y negación en magnitudes: cantidades o grados de determinidad (activa o pasiva). Esto anula la diferencia que hacía posible la contradicción. Una vez cuantificadas, realidad y negación devienen magnitudes cualitativamente iguales: "un grado", dice Fichte, "es siempre un grado, sea de realidad o de negación."59

\footnotetext{
56 GWL, GA I/2, 287-288.

57 GWL, GA I/2, 261, 267, y 282.

$58 \mathrm{GWL}, \mathrm{GA}$ I/2, 288.

59 GWL, GA I/2, 289.
} 
Los grados de realidad y de negación que puedan predicarse de Yo y No-Yo representan las posibles divisiones en las que la totalidad de la consciencia puede determinarse. Cada determinación de Yo y No-Yo (aquello que a nivel consciente es una representación concreta) es, pues, un modo de dividir el todo de la realidad en la consciencia. Esta totalidad es, según Fichte, un "quantum absoluto". ${ }^{60}$ Esto significa: i) que no hay un afuera de la consciencia; ii) que, por ende, realidad y negación poseen un sentido distinto, pero un mismo significado, dado que ambas refieren al contenido de toda consciencia posible; iii) que la totalidad de los contenidos posibles de consciencia es una cantidad invariable, idéntica consigo misma; iv) que, en consecuencia, la relación que se da en cada determinación (sea del Yo o del No-Yo) entre realidad y negación es inversamente proporcional. 61 "De acuerdo con esto", dice Fichte, "el Yo pone negación en sí mismo en la medida en que pone realidad en el No-Yo, y realidad en sí mismo en la medida en que pone negación en el No-Yo".62 En consecuencia, la cuantificación de la cualidad hace que toda limitación entre realidad y negación sea de por sí recíproca.

La cuantificación de la realidad es, empero, el momento último de la primera síntesis. En efecto, quedan aún dos pasos para completar esta primera síntesis, los cuales corresponden con aquello que produce la limitación recíproca entre Yo y NoYo. El primero consiste en determinar en general aquello que une las acciones de cuantificar y limitar (dividir en grados) la totalidad del contenido de toda consciencia posible. El segundo paso, es deducir el carácter propio de esta determinación en cuanto síntesis originaria de cualidad y cantidad.

La limitación (posición simultánea de realidad y negación en reciprocación ${ }^{63}$ ) y la cuantificación del todo de la consciencia son según la GWL dos caras de una misma acción sintética originaria del Yo, la cual está contenida en el "género [Gattungsbegriff] superior de la determinación [Bestimmung]".64 El concepto de determinación, sin embargo, no define exhaustivamente la síntesis acontecida, pues, tal como Fichte aclara, no muestra "la diferencia específica"65 que distingue esta acción de la acción de determinar en general.

La limitación entre Yo y No-Yo que la posición de la cantidad hace posible, es, como se ha dicho, necesariamente recíproca. La primera acción sintética en la deducción de las categorías no es, por lo tanto, "determinación en general" 66 , sino

\footnotetext{
60 GWL, GA I/2, 288.

61 GWL, GA I/2, 288-289.

62 GWL, GA I/2, 289.

63 En la traducción de "Wechsel" por "reciprocación" se sigue la sugerencia de J. Cruz Cruz en su traducción de la GWL. (Fundamento de toda la doctrina de la ciencia (1794), p. 88)

64 GWL, GA I/2, 289.

65 Ibídem.

66 Ibídem.
} 
posición o determinación de la cantidad de un modo determinado. Este modo determinado consiste en que:

la cantidad de uno es puesta a través de la cantidad de su opuesto y viceversa. A través de la determinación de la realidad o negación del Yo es determinada a la vez la negación o realidad del No-Yo y viceversa. ${ }^{67}$

De allí que esta síntesis no sea mera determinación, sino determinación recíproca, la cual según Fichte es "lo mismo que en Kant se llama relación."68

Dejando de lado la cuestión acerca de si aquello que Kant llama relación coincide con la determinación recíproca fichteana, resulta importante atender a lo que esta afirmación sugiere, lo cual, por cierto, se volverá evidente en los pasos siguientes de la deducción. La identificación entre "relación" y "determinación recíproca" significa que con la deducción de esta última ha sido deducido a la vez el grupo todo de la relación. En efecto, como habrá de verse, causalidad y sustancialidad son para Fichte modos de la determinación recíproca.

A la identificación que subordina causalidad y sustancialidad a la determinación recíproca, se suman dos identificaciones más en esta primera síntesis. Primero, la identificación de la limitación con la determinación recíproca, dado que, de acuerdo con el carácter invariable de la totalidad, toda limitación entre Yo y No-Yo es recíproca. ${ }^{69}$ Segundo, la identificación de la cantidad con la determinación recíproca mediante la identificación entre cantidad y limitación. ${ }^{70}$ Estas identificaciones son, por cierto, sólo parciales, pues en cada caso el correlato de la determinación recíproca no coincide con la totalidad de lo que esta categoría o acción sintética denota. Ellas muestran, sin embargo, el modo en que Fichte articula internamente el conjunto categorial: la determinación recíproca es la categoría primera, pues la deducción de las categorías restantes debe presuponerla en cuanto acción ya realizada.

El primer acto sintético en sentido estricto corresponde, entonces, con la categoría de determinación recíproca. Sólo mediante ella es resuelta completamente la primera contradicción del teorema del saber teórico. A través de esta primera síntesis han sido deducidos además los grupos de la cuantidad y cualidad in concreto y el grupo de la relación in abstracto.

El encadenamiento causal descrito hasta aquí se da del siguiente modo: i) el Yo pone la determinación recíproca como síntesis de la contradicción entre las dos pro-

\footnotetext{
67 GWL, GA I/2, 290.

68 Ibídem.

69 La limitación es, entonces, como Fichte advierte en sus Meditaciones personales, manuscrito de 1793/94, una "limitación interna que se limita a sí misma", la cual es "sin ninguna duda efectuación recíproca.” (GA II/3, 45).

70 La limitación es definida como "un poner de cantidad en general". (GWL, GA I/2, 282).
} 
posiciones que el teorema del saber teórico contiene; ii) esta acción sintética implica dos momentos: poner la totalidad de la realidad en términos cuantitativos (categoría de cantidad) y determinar realidad y negación de Yo y No-Yo en la consciencia (limitación recíproca de ambos en el Yo). En este sentido, el grupo de la cualidad es efecto del de la cantidad y ambos son productos de la síntesis de determinación recíproca.

$* * *$

Esta primera síntesis resuelve ciertamente la contradicción entre las dos proposiciones contenidas en el teorema del saber teórico: "el Yo se pone como determinado por el No-Yo" y "el Yo se determina". Sin embargo, al inicio del apartado B del $\S 4$, en el que se deduce la síntesis de determinación recíproca, Fichte advierte que no debe descartarse la posibilidad de que "cada una de ambas proposiciones esté en contradicción consigo misma". ${ }^{71} \mathrm{El}$ paso siguiente de la deducción fichteana consiste precisamente en la exposición y solución de las contradicciones que cada una de estas proposiciones contiene por separado. Este despliegue de antítesis y síntesis tiene como correlato la diferenciación interna de la determinación recíproca en causalidad y sustancialidad (deducción del grupo de la relación in concreto).

\subsection{Segunda síntesis: causalidad, realidad y negación positivas (hacer y padecer), momento cuantitativo de la particularidad}

La primera de las proposiciones contenidas en el teorema del saber teórico reza "el Yo se pone como determinado por el No-Yo". Su demostración (deducción) significa explicar uno de los momentos de la determinación recíproca, a saber: "cómo el Yo puede poner negación en sí mismo y realidad en el No-Yo".72 Esta pregunta es respondida mediante la deducción de la síntesis (categoría) de causalidad. Ella explica cómo es posible predicar actividad de aquello que según la proposición de la espontaneidad absoluta del Yo, a saber, "el Yo es fuente de toda realidad", 73 debería ser considerado como no activo (el No-Yo).

La deducción de la segunda síntesis originaria se inicia con la identificación de la contradicción contenida en la proposición "el Yo se pone como determinado por el No-Yo" y la demostración de que esta contradicción debe ser resuelta. Esto último significa que, como en la síntesis anterior, la contradicción es sólo aparente, pues el factum de la realidad de consciencia y autoconsciencia empíricas implican

\footnotetext{
71 GWL, GA I/2, 287.

72 GWL, GA I/ 2, 289

73 GWL, GA I/2, 293.
} 
que la misma ya ha sido resuelta en la génesis real que la doctrina de la ciencia busca reconstruir. 74

La proposición "el Yo se pone como determinado por el No-Yo" supone que "el No-Yo debe determinar el Yo", lo cual contradice la premisa "toda realidad es puesta en el Yo". Esta premisa no se asienta sólo en el carácter absoluto del Yo, el cual es evidente para Fichte, sino además en lo que se ha deducido en la síntesis anterior, a saber: que mediante la cuantificación de la cualidad el Yo ha puesto en la consciencia la totalidad de la realidad en general (en cuanto ser algo y no otra cosa o nada, 75 es decir limitación de realidad y negación).

Por un lado, entonces, se sostiene, que el No-Yo tiene realidad en sí y que, dado el carácter invariable de la totalidad, esta realidad corresponde con una anulación de realidad de igual magnitud en el Yo. Por el otro, se afirma que la realidad no puede ser puesta en otro miembro que no sea el Yo ni puede tener como origen en sí algo que no sea el Yo y que el No-Yo es, por lo tanto, negación sin más y no puede tener en sí realidad. 76

Al igual que en la síntesis anterior, luego de exponer la contradicción correspondiente, Fichte aclara que esta proposición ha sido deducida necesariamente del teorema del saber teórico. Por consiguiente, la proposición no puede anularse y las afirmaciones opuestas contenidas en la misma deben ser unificadas, pues, de otro modo, se anularía "la unidad de la consciencia en la que la proposición capital [sc. el tercer axioma] está contenida”. Esta anulación implicaría además la anulación del teorema del saber teórico y, por consiguiente, de la primera síntesis ya deducida. ${ }^{77}$

Lo que la síntesis de causalidad debe explicar es cómo es posible quitar partes de realidad del Yo y trasladarlas al No-Yo sin que el carácter del Yo de ser "fuente de toda realidad" sea anulado. El primer paso consiste en una nueva determinación de realidad y negación, puesto que la relatividad que habían adquirido mediante su cuantificación impide establecer la diferencia entre Yo y No-Yo exigida tanto por la evidencia de la consciencia empírica (la distinción interno/externo) como por el postulado de la espontaneidad del Yo. ${ }^{78}$

La nueva determinación de realidad y negación consiste en pensar estos conceptos en términos positivos de acción y pasión: hacer y padecer como modos del hacer en general.

El Yo debe ser determinado, esto es, realidad o, como este concepto ha sido determinado, actividad debe ser anulada en él. Por consiguiente es puesto en él lo contrario de la

\footnotetext{
74 GA I/2, 140-141 Über den Begriff der Wissenschaftslehre y GA I/4, 209-210 Zweite Einleitung in die Wissenschaftslehre.

75 Janke, W., Fichte. Sein und Reflexion, Berlin: De Gruyter, 1970, p. 132.

76 GWL, GA I/2, 291.

77 Ibídem.

78 GWL, GA I/291-292.
} 
actividad. Lo contrario de la actividad, empero, se llama padecer. Padecer es negación positiva y en tal medida está contrapuesta a la [negación] meramente relativa. 79

La determinación de realidad y negación en cuanto positivas surge de proyectar la identidad ser=poner=ser activo que se predica del Yo sin más al todo de la realidad en la consciencia (Yo y No-Yo divisibles). ${ }^{80}$ El carácter positivo de realidad y negación no cancela la determinación cuantitativa de la cualidad, pues el carácter relativo de ésta (ser magnitud/diferencia de un mismo género) está presente en la ausencia de una diferencia cualitativa entre hacer y padecer en cuanto modos del hacer en general. La diferencia cualitativa se da en la oposición entre actividad y reposo en cuanto grado 0 de actividad. 81

La determinación del Yo por medio del No-Yo consiste en predicar del No-Yo un hacer como correlato de un padecer en el Yo. Padecer, dice Fichte, es "afección [Affektion] en general": 82 algo que no puede ser referido inmediatamente a la actividad del Yo. De acuerdo con el carácter constante/invariable de la totalidad de la realidad, al estado de padecer del Yo debe corresponderle por determinación recíproca el mismo grado de actividad en el No-Yo. En el padecer del Yo, entonces, está ya contenido el actuar del No-Yo. El padecer del Yo, luego, es lo que permite predicar realidad (positiva) del No-Yo.

El No-Yo en cuanto tal no posee en sí realidad alguna; pero posee realidad en la medida en que el Yo padece, en virtud de la ley de determinación recíproca. [...] el No-Yo posee $[. .$.$] realidad para el Yo sólo en la medida en que el Yo es afectado y fuera de la$ condición de una afección del Yo no posee ninguna en absoluto. ${ }^{83}$

La realidad es, entonces, transferida (übertragen) del Yo al No-Yo mediante el padecer del Yo. Esta transferencia es, según Fichte, el significado originario de la categoría de causalidad. ${ }^{84}$

La determinación de Yo y No-Yo en la síntesis de causalidad, por lo tanto, se inicia con el padecer del Yo. Esta afección es un efecto que en cuanto tal remite a un actuar del No-Yo como a su contrario necesario: la causa. Esta referencia causal, empero, se da en la inmanencia de la consciencia. Toda la realidad, luego, sigue

\footnotetext{
79 GWL, GA I/2, 293.

80 Ibídem.

81 Ibídem. La oposición actividad/reposo juega, por cierto, un rol fundamental al inicio de la Doctrina de la ciencia nova methodo. Allí, sin embargo, esta oposición no es pensada en términos cualitativos, sino cuantitativos, lo cual permite a Fichte afirmar que el No-Yo es un modo de considerar al Yo. (GA IV/ 2, 32 y ss. Nachschrift Halle, GA IV/3,348 y ss., Nachschrift Krause).

82 GWL, GA I/2, 293.

83 GWL, GA I/2, 294.

84 Ibídem.
} 
estando puesta en el Yo: una parte como padecer del Yo y otra parte como hacer del No-Yo. El ser afectado del Yo no implica, entonces, que éste deje de ser fuente de toda realidad. Pues el padecer no es correlato de una realidad en sí fuera del Yo, sino de una realidad proyectada por el Yo en el No-Yo. Por consiguiente, la síntesis de la causalidad no postula una realidad en sí del No-Yo. El No-Yo posee realidad sólo en la medida en que el Yo padece. Fuera de la condición del padecer, el No-Yo, según la GWL, no posee realidad alguna. Por consiguiente, la realidad del No-Yo es sólo para el Yo.

La síntesis de causalidad establece un nuevo modo de relación recíproca entre Yo y No-Yo en el que el primero es entendido como efecto del segundo. Ambos, efecto y causa, componen una misma realidad entendida como "efectuación" (Wirkung). ${ }^{85}$ En ella la causa (Ursache) no antecede al efecto (Effekt), sino que ambos se dan a la vez. En este sentido, Fichte señala que

en absoluto es cierto que se tenga que pensar la causa, en cuanto tal, esto es en la medida en que ella es activa en la efectuación determinada, como precediendo en el tiempo a lo efectuado [dem bewirkten]. 86

La síntesis de causalidad, según Fichte, abstrae, pues, del tiempo como condición para pensar y determinar esta relación. La necesidad de esta abstracción aparece primero como condición para pensar de modo puro la actividad ${ }^{87} \mathrm{y}$, luego, como condición para pensar la unidad sintética de causa y efecto en la efectuación: "causa y efectuado deben por cierto ser pensados en cuanto uno y precisamente lo mismo en virtud de la unidad sintética". 88

Para este segundo caso Fichte ofrece dos argumentos. Por un lado, el tiempo en general no ha sido aún deducido, lo cual no autoriza su introducción; 89 por otro, el esquema de la sucesión temporal no debe aplicarse a los elementos de la efectuación en cuanto causa y efecto, sino en cuanto sustancias (substratos de cualidades). 90 De allí que en la GWL la deducción de las categorías y de sujeto y objeto en sentido teórico (substratos) anteceda a la del tiempo.91

\footnotetext{
85 Ibídem.

86 GWL, GA I/2, 293-294.

87 GWL, GA I/2, 293.

88 GWL, GA I/2, 294.
}

89 Este argumento pierde fuerza, cuando se advierte que al inicio de la síntesis E, Fichte recurre al concepto de tiempo, sin aún haberlo deducido, para explicar el problema de la circularidad del sistema categorial. (GWL, GA I/2, 302-303)

$90 \mathrm{GWL}, \mathrm{GA} \mathrm{I} / 2,295$. Las primeras versiones de la deducción de las categorías en el manuscrito Eigne Meditationen über ElementarPhilosophie muestran, sin embargo, que Fichte en un comienzo introducía el tiempo en el tratamiento de causalidad y substancialidad (GA II/3, 72, 78 y ss.).

${ }^{91}$ La deducción de tiempo y espacio en la GWL se limita sólo a consideraciones fundamentales. Fichte presenta una deducción genética de tiempo y espacio sensu stricto por primera vez en Grundriss des 
Ahora bien, mientras en el primer caso señalado Fichte sostiene que se debe abstraer "de todas las condiciones temporales [von allen Zeitbedingungen]", 92 en el segundo, al analizar la unidad entre causa y efecto, Fichte considera que de lo que se debe abstraer es "de las condiciones empíricas del tiempo [von den empirischen Zeitbedingungen]". ${ }^{93}$ En este sentido cabe preguntar acerca del alcance del adjetivo 'empírico' en el segundo caso. Atendiendo a lo que Fichte sostiene en el párrafo en que aparece el adjetivo 'empírico', podría conjeturarse que "todas las condiciones temporales" se refieren al tiempo sin más, esto es, en cuanto forma de la intuición, que en este momento de la deducción no ha sido aún deducido, mientras que las "condiciones empíricas del tiempo" son aquí un modo de llamar al tiempo en cuanto sucesión concreta de distintos estados referidos a dos substancias también concretas. Todo parece indicar, sin embargo, que esta especificación de las condiciones temporales en cuanto empíricas no agrega nada nuevo en este pasaje con respecto a lo que significa "condiciones temporales" en el primer caso. Tanto en el caso de la actividad como en el de la relación de causalidad se debe abstraer, según Fichte, del tiempo en cuanto aquello que "no ha sido aún deducido"94, lo cual refiere tanto a determinaciones empíricas temporales como al tiempo en cuanto forma pura de la intuición sensible.

La categoría de causalidad es deducida en la GWL como una especificación de la determinación recíproca, pues en ella ya no es indiferente en qué miembro de la reciprocación son puestas realidad y negación. La distinción entre causa y efecto indica que la reflexión ya no se mueve en el momento cuantitativo de la totalidad, sino que se concentra en el de la particularidad. La deducción de la causalidad explica, sin embargo, sólo en qué consiste el hecho de que el Yo sea determinado por el No-Yo. Queda aún por explicar cómo es posible que el Yo se ponga como determinado por el No-Yo. Esta es la tarea que resuelve la tercera síntesis.

\footnotetext{
Eigenthümlichen der Wissenschaftslehre in Rücksicht auf das theoretische Vermögen (1795) (GA I/3, 193-208, §4). Véase además Metz, W., "Fichtes genetische Deduktion von Raum und Zeit in Differenz zu Kant", Fichte-Studien 6 (1994), pp. 71-94 y Acosta, E., "The Deduction of Time and Space in J.G. Fichte's Theory of the Co-Constitution of Subject and Object", International Journal of Computing Anticipatory Systems, 26 (2014), pp. 69-78. Acerca de la deducción de espacio y tiempo en la Wissenschaftslehre nova methodo, véase Serrano M., V., "La deducción del espacio y el tiempo en los parágrafos 10 a 12 de la Doctrina de la ciencia nova método del manuscrito de Krause", Endoxa, 30 (2012), pp. 255-272.

92 GWL, GA I/2, 293.

93 GWL, GA I/2, 294.

94 Ibídem.
} 


\subsection{Tercera síntesis: sustancialidad, realidad y negación determinadas y determinables (relatividad de hacer y padecer) y momento cuantitativo de la unidad}

El último paso en la deducción de las categorías se inicia con el análisis de la segunda proposición del teorema del saber teórico, dejada en suspenso en la síntesis anterior: "el Yo se pone en cuanto determinado, esto es, se determina". ${ }^{55}$ Lo que debe explicarse aquí es cómo es posible la acción del Yo de ponerse (como determinado por el No-Yo). La contradicción a resolver consiste en que el Yo es descripto en esta proposición como lo determinante "y por lo tanto activo" y a la vez como "lo que es determinado y por lo tanto pasivo". 96 Siguiendo a Fichte, se debe deducir un nuevo concepto sintético que permita pensar la compatibilidad de activo y pasivo ya no en la reciprocación entre Yo y No-Yo, sino en el Yo mismo tomado como unidad. Para esto Fichte introduce primero una nueva determinación de realidad y negación, la cual se articula en tres pasos: análisis de la relación todo/parte, identificación entre realidad y actividad, e identificación de hacer y padecer determinados en contraposición a la totalidad del hacer en cuanto determinable.

Al igual que en la causalidad, la síntesis de sustancialidad comienza con la apelación a la idea del Yo como "totalidad absoluta de la realidad". ${ }^{97}$ A diferencia de la síntesis de causalidad el análisis se concentra ahora en la relación entre todo y parte mentada en esta idea. En esta relación cada parte es a la vez afirmación y negación del todo: afirmación, pues la parte se identifica con el todo en cuanto parte del mismo, y negación, pues ella se diferencia del todo en cuanto negación de las partes restantes que también componen la totalidad. ${ }^{98}$

Una vez establecidas estas distinciones, Fichte vuelve a introducir, como en la síntesis de causalidad, la identificación entre realidad y actividad tal como aparece en la idea del Yo como lo activo sin más. De esta identidad Fichte concluye primero la identidad de lo no-activo con el No-Yo y, luego, el carácter relativo del padecer con respecto a la actividad, esto es, que el padecer no es sino un no-actuar. 99

Este carácter relativo, sostiene Fichte, supone que hay algo que comunica hacer con padecer: la cantidad. Por lo tanto, el padecer debe ser considerado como " $u n$ quantum de actividad." 100 A partir de las distinciones referidas a la relación entre todo y parte, entonces, Fichte concluye que una parte de actividad es a la vez nega-

\footnotetext{
95 GWL, GA I/2, 295.

96 Ibídem.

97 GWL, GA I/2, 296.

98 GWL, GAI/2, 296.

99 GWL, GA I/2, 296-297.

100 GWL, GA I/2, 297.
} 
ción (padecer) y afirmación (hacer) de la totalidad del actuar. Esta totalidad en cuanto determinabilidad (conjunto de las posibilidades en que todo hacer y padecer puede darse) sirve de criterio de medida para todo hacer o padecer determinado. La identidad y diferencia que Fichte establece aquí entre hacer y padecer radica entonces en el carácter de determinado. Hacer y padecer concretos se identifican en cuanto determinados. Esta identificación es posible mediante la contraposición de ambos con la totalidad del actuar en cuanto lo determinable sin más.

De este modo queda resuelta la contradicción en la segunda proposición del teorema teórico. El Yo es determinante y determinado a la vez pues la acción de ponerse es un actuar determinado que en relación con el No-Yo sin más es hacer (afirmación del carácter activo del Yo) y en relación con la totalidad del hacer (la suma de todas las actividades del Yo) es, "aun cuando sea en sí actividad", padecer (negación del carácter del Yo de ser potencialmente la totalidad del hacer). ${ }^{101}$ Realidad y negación son, pues, deducidas como determinabilidad y ser determinado mediante la relativización del par conceptual hacer/padecer.102

Según la síntesis de sustancialidad el Yo en cuanto conteniendo todas las realidades posibles (determinabilidad del actuar) es substancia y en cuanto actuar determinado es accidente. El Yo resulta una X que "es realidad, negación, actividad y padecer a la vez". ${ }^{103}$ Lo importante aquí es la relación entre substancia y accidente que se deduce de esta síntesis: ninguno puede ser pensado sin el otro. La substancia está compuesta por la totalidad de los accidentes, y el accidente es sólo tal en comparación con una totalidad que en sentido estricto corresponde con una unidad en cuanto substrato de realidades determinables. 104

\section{El sistema fichteano de las categorías: una tríada de tríadas bajo el prima- do de la determinación recíproca}

La síntesis de sustancialidad resuelve la última contradicción interna del teorema del saber teórico y, en este sentido, marca el final de la deducción de las categorías en la GWL. La deducción fichteana de las categorías consiste entonces en (re)construir las síntesis de las antítesis contenidas en este teorema. La síntesis de determinación recíproca resuelve la contradicción entre las dos proposiciones que componen el teorema. La síntesis de causalidad resuelve la contradicción entre las afirmaciones contenidas en la primera de las proposiciones. La síntesis de sustan-

\footnotetext{
101 Ibídem.

102 Klotz, Ch., "Der Ichbegriff in Fichtes Erörterung der Substantialität", Fichte-Studien 10 (1997), p. 161.

103 GWL, GA I/2, 297.

104 GWL, GA I/2, 299-300.
} 
cialidad, por último, explica cómo se concilian las afirmaciones contenidas en la segunda proposición del teorema. 105

En estas tres síntesis son deducidas además las categorías de cualidad y cantidad, las cuales son ingresadas mediante la primera síntesis y luego en las dos síntesis restantes adquieren nuevas determinaciones. La deducción estructura, entonces, el sistema categorial como una tríada de tríadas, la cual consiste en un conjunto cerrado. Esto es de verse en la circularidad que se origina cuando Fichte explica la dinámica del sistema categorial.106 De allí que el paso siguiente en la GWL sea la postulación de dos elementos externos a la reciprocación entre Yo y No-Yo en el Yo: la actividad independiente y el choque (Anstoss). ${ }^{107}$

Dentro del conjunto categorial la deducción establece nexos causales que implican relaciones de dependencia y una jerarquía entre las categorías. En primer lugar, la deducción identifica cada síntesis con una categoría de relación en particular, lo cual muestra la primacía de la relación sobre la cualidad y la cantidad.108 En segundo lugar, se da un ordenamiento jerárquico dentro del grupo de la relación. Las dos últimas síntesis son especificaciones de la primera. 109 Causalidad y sustancialidad son, luego, conceptos sintéticos "contenidos en el concepto superior de determinación recíproca." 110 Esto muestra el primado de la categoría de determinación recíproca dentro del grupo de la relación. En tercer lugar, el surgimiento de las categorías de cualidad y cantidad se da mediante la primera síntesis, lo cual afirma el carácter central en cuanto primera categoría de la categoría de determinación recíproca. Fichte transforma así la tabla cuádruple kantiana en una tríada de tríadas bajo el primado de la determinación recíproca:

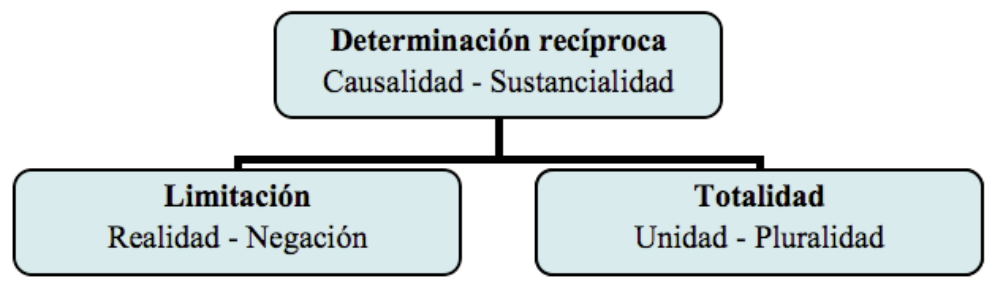

La determinación recíproca en cuanto categoría principal de la tabla fichteana de las categorías representa la síntesis (acción originaria) principal dentro del des-

\footnotetext{
105 Ibídem.

106 GWL, GA I/2, 302-303.

107 GWL, GA I/2, 304-306 y 355, respectivamente.

108 GA IV/3, 485 Fichtes Vorlesungen über die Wissenschaftslehre, gehalten zu Jena im Winter 1798/99, Nachschrift Krause.

109 GWL, GA I/2, 290.

110 GWL, GA I/2, 291 y 299.
} 
pliegue del teorema del saber teórico. En cuanto tal, sin embargo, ella no debe ser identificada con el principio del sistema todo (el Yo absoluto). Junto con las síntesis de causalidad y sustancialidad, y por ende junto con los grupos categoriales de la cualidad y la cantidad, la determinación recíproca se halla doblemente subordinada. En primer lugar, se subordina a la síntesis originaria que expresa el tercer axioma, dado que surge de una reflexión sobre el mismo. En segundo lugar, siguiendo la estructura del sistema, se halla subordinada al Yo en cuanto sujeto absoluto, el cual, como se ha dicho en (3.2), exige y produce la síntesis originaria y las síntesis restantes. La presente reconstrucción coincide, pues, con la indicación de Fichte de que: "aquello de lo cual toda categoría es deducida [es] el Yo, en cuanto sujeto absoluto."111

Fichte reúne así en un mismo concepto (el del Yo en cuanto sujeto absoluto) el principio del sistema todo y el de las categorías, reformulando, o bien ampliando, el concepto kantiano de apercepción pura. Fichte cree así estar llevando a cabo aquello que Kant sólo se limito a indicar pero no a demostrar:

En su deducción de las categorías Kant ha señalado nuestra proposición [sc. Yo soy en cuanto expresión de la Tathandlung] en cuanto axioma absoluto de todo saber, pero no lo ha establecido nunca de modo determinado en cuanto axioma. 112

La apercepción pura es identificada en la GWL con la acción originaria de la Tathandlung (literalmente: acción-hecho) que la proposición 'Yo soy' denota.113 Con esta resignificación, empero, Fichte no desconoce el contenido original del concepto kantiano, sino que lo ubica, al igual que las categorías kantianas, 114 en un nivel inferior: el de los hechos de consciencia. En efecto, la apercepción pura kantiana entendida como el "Yo pienso" que "tiene que poder acompañar todas mis representaciones"115 corresponde con lo que Fichte en el $\S 1$ de su GWL llama el 'Yo soy' "en cuanto expresión [...] de un hecho [Thatsache])". 116 A diferencia del 'Yo soy' en cuanto Tathandlung (sujeto absoluto), este 'Yo soy' tiene que poder acompañar todo hecho de consciencia. Si bien este 'Yo soy' es 'el hecho supremo de la consciencia empírica", se halla, al igual que la apercepción pura kantiana, en interdependencia con aquello de lo cual es condición de posibilidad (los hechos de consciencia en general). ${ }^{117}$

\footnotetext{
111 GWL, GA I/2, 262.

112 Ibídem. Einleitung in die Wissenschaftslehre, 1797).

114 GA I/4, 201, Erste Einleitung in die Wissenschaftslehre.

$115 \mathrm{KrV}$ B 131-132.

116 GWL, GA I/2, 257.

117 GWL, GA I/2, 258.
}

113 Esta reformulación de la apercepción pura kantiana Fichte la expresa en términos más claros en escritos posteriores al periodo que aquí se estudia. (Véase por ejemplo GA I/4, 225 y 255-256, Zweite 
La dinámica de la deducción fichteana de las categorías en la GWL no sólo establece la subordinación de todas las categorías a la de determinación recíproca. Con cada síntesis, por un lado, las categorías de realidad y negación adquieren una nueva determinación, y, por otro, un momento de la cantidad es puesto en el centro del análisis:

\begin{tabular}{|l|l|l|}
\hline Relación & Cualidad & Cantidad \\
\hline $\begin{array}{l}\text { Determinación } \\
\text { recíproca }\end{array}$ & Realidad y negación relativas & $\begin{array}{l}\text { Totalidad de contenidos } \\
\text { posibles de consciencia }\end{array}$ \\
\hline Causalidad & $\begin{array}{l}\text { Realidad y negación positivas (hacer y } \\
\text { padecer) }\end{array}$ & $\begin{array}{l}\text { Pluralidad/diversidad: ser } \\
\text { causa o efecto }\end{array}$ \\
\hline Sustancialidad & $\begin{array}{l}\text { Realidad en cuanto determinabilidad y } \\
\text { realidad y negación determinadas } \\
\text { (relatividad de hacer y padecer) }\end{array}$ & $\begin{array}{l}\text { Lo uno como substrato de } \\
\text { realidades }\end{array}$ \\
\hline
\end{tabular}

La sistematización fichteana de las categorías implica la exclusión del grupo de la modalidad y la anulación de la distinción entre categorías matemáticas y dinámicas. Según lo expuesto en (2), para Fichte las categorías dinámicas son en la GWL categorías en sentido secundario o "en el más bajo de sus niveles": conceptos puros del entendimiento ya aplicados a un objeto dado potencial o efectivamente. ${ }^{118} \mathrm{La}$ modalidad pertenece en la GWL a este grupo de categorías. Las categorías modales son deducidas como modos en que el objeto se presenta al sujeto en la representación. De allí que su lugar en el sistema sea el de la Deducción de la representación 119 y no el de la deducción de las síntesis originarias de las que, por cierto, surgen los elementos para explicar la representación. 120 La modalidad no explica pues cómo surge la representación, sino los modos en que se da la aprehensión pre-consciente del objeto (en sentido teórico) en general.

Las categorías en sentido estricto refieren, por el contrario, a acciones originarias del Yo anteriores al surgimiento de las formas puras de la sensibilidad y de sujeto y objeto teóricos. La determinación de Yo y No-Yo en cuanto, respectivamente, sujeto y objeto teóricos, supone la diferenciación de Yo y No-Yo en cuanto puestos dentro (sujeto y objeto) y fuera (actividad independiente y choque) de la reciprocación. ${ }^{121}$ La deducción de las formas puras de la sensibilidad, tiempo y espacio, viene exigida en la GWL en función de la determinación de Yo y No-Yo como sujeto y objetos teóricos. La idealidad del tiempo, según Fichte, se deduce de la idealidad de objeto y sujeto. ${ }^{122} \mathrm{El}$ tiempo aparece en la GWL como producto de la ima-

118 GA I/4, 201, Erste Einleitung in die Wissenschaftslehre.

119 GWL, GA I/2, 374, 378.

120 GWL, GA I/2, 362.

$121 \mathrm{GWL}, \mathrm{GA} \mathrm{I} / 2,356$ y ss.

122 En GWL, GA I/2, 335 n. Fichte sostiene que, a diferencia de Kant, la doctrina de la ciencia demuestra "la idealidad del tiempo a partir de la idealidad de los objetos". Se incluye aquí la idealidad del sujeto, primero, a partir de lo que sostiene Fichte una páginas más atrás, a saber, que "sin sujeto no 
ginación en su oscilar entre sujeto y objeto teóricos y entre las distintas determinaciones que recibe el Yo. En este sentido Fichte sostiene que "sólo para la imaginación hay tiempo". ${ }^{123}$ En el ámbito de las acciones originarias del Yo no hay tiempo ni espacio. De allí que Fichte exija, como se ha indicado en (3.4), que causa y efecto sean pensados como simultáneos.

\section{Dificultades de la lectura habitual de la deducción fichteana de las categorías}

En la sección anterior se ha mostrado que el sistema categorial fichteano consiste en un sistema de tres tríadas de categorías en el que la categoría de determinación recíproca resulta la categoría fundamental. Salvo contadas excepciones que reconocen la primacía de esta categoría, ${ }^{124}$ la lectura habitual de la deducción de las categorías en la GWL confunde la secuencia en que las categorías aparecen en este escrito con el orden sistemático en que efectivamente son deducidas. ${ }^{125}$ Según esta lectura la deducción de las categorías se inicia con las categorías de cualidad (§§13 ), a las que siguen la cantidad ( 3 ), el grupo de la relación ( $\$ 4$ B-D) y, por último, las categorías de modalidad ( $§ 4$, Deducción de la representación). Esta interpretación no repara, empero, en los problemas conceptuales y metodológicos que ella misma ocasiona.

Según esta lectura los cuatro grupos categoriales son deducidos en tres secciones distintas del escrito, las cuales corresponden con niveles diferentes del sistema. Así, realidad y negación son deducidas antes de la síntesis fundamental de la GWL y limitación y cantidad, antes del establecimiento del teorema del saber teórico; mientras que el grupo de la relación es deducido a partir de este teorema, y el de la modalidad, sólo una vez que objeto y sujeto teóricos han sido determinados. La supuesta deducción, entonces, obtiene cada grupo categorial a partir de un principio diferente: las acciones originarias de poner, contraponer y limitar para la cualidad y

hay objeto, sin objeto no hay sujeto" (GWL, GA I/2, 332). Por otra parte, se debe atender al hecho de que el tiempo surge a través del oscilar de la imaginación entre opuestos de por sí irreconciliables (Yo y No-Yo en sentido estricto) y que este surgimiento del tiempo supone la determinación de los opuestos ya no como Yo y No-Yo sin más, sino en cuanto sujeto y objeto respectivamente. (GA GWL I/2, 357, 360-361)

123 GWL, GA I/2, 360.

124 Kraus, E., Der Systemgedanke bei Kant und Fichte, Erganzungsheft der Kantstudien 37, Berlin, 1916, pp. 55 y s.; y Jürgensen, S., Freiheit in den Systemen Hegels und Schellings, Wurzburg: Köngshausen \& Neumann, 1997, p. 33.

125 Medicus, C., J.G. Fichte. Dreizehn Vorlesungen, Berlin: Reuther und Reichard, 1905, p. 92; Janke, W., Sein und Reflexion, pp. 13 y 132; Cesa, C., Fichte e l'idealismo trascendentale, Bologna: Il Mulino 1992, p. 29; Marquard, O., Theodizeemotive in Fichtes früher Wissenschaftslehre, Erlangen/Jena: Palm \& Enke, 1994, p. 29; Riedel, Ch., Zur Personalisation des Vollzuges der Wissenschaftslehre J.G. Fichtes, Stuttgart: Franz Steiner, 1997: 21; Kubo, Y., "Transformation der Deduktion der Kategorien Fichte und Hegel”, Fichte-Studien 21 (2003), p. 79. 
la cantidad, el teorema del saber teórico para la relación y la representación para la modalidad.

Tampoco queda claro en este modo de leer la deducción fichteana de las categorías cuál es el método de obtención de las categorías. Pues siguiendo esta interpretación se debe concluir que, si bien a partir de principios diferentes, sólo la relación y la modalidad ${ }^{126}$ son en verdad deducidas genéticamente, mientras que las categorías de cualidad son obtenidas mediante abstracción 127 (lo cual, como se ha expuesto en (2), Fichte critica de Kant) y la cantidad es ingresada sin más como sinónimo de limitación. ${ }^{128}$ A esto debe añadirse el inconveniente que trae la inclusión de la modalidad en el conjunto de las categorías en cuanto acciones originarias del Yo: este grupo es deducido mediante la presuposición del objeto en cuanto ya dado, lo cual, como se ha expuesto en (2), Fichte critica de la deducción kantiana.

Esta interpretación de la GWL lleva, además, a sostener la primacía de la categoría de realidad, dado que en ella, se dice, "se fundan las demás categorías". 129 Esta tesis argumenta exclusivamente a partir de citas tomadas aisladamente en las que Fichte parece sugerir un vínculo exclusivo entre la categoría de realidad y la realidad absoluta del Yo. ${ }^{130}$ En la sección anterior se ha mostrado que ni la categoría de realidad es el fundamento del conjunto categorial ni la cualidad es el primer grupo en ser deducido.

En primer lugar, como se ha mostrado, la categoría de realidad supone las de negación y limitación, de allí que realidad no pueda ser la categoría fundamental. Realidad y negación en cuanto categorías son concebibles, según Fichte, sólo a partir de la limitación: sólo por medio de esta categoría puede predicarse realidad y negación acerca de algo. ${ }^{131}$

En segundo lugar, el vínculo entre las categorías de realidad y negación y las acciones originarias de poner y contraponer en los $\S \S 1$ y 2 de la GWL no implica la primacía de estas categorías. Pues, realidad y negación no son deducidas en estos parágrafos, sino obtenidas mediante abstracción, lo cual no dice nada acerca del lugar dentro del sistema categorial que ellas reciben al ser generadas. Se ha visto en (2) que Fichte deduce ambas categorías junto con la limitación a partir de la determinación recíproca.

En tercer lugar, la realidad y la negación del poner y contraponer originarios no corresponden con las categorías homónimas. 132 Por un lado, mientras las categorí-

\footnotetext{
126 Cf. GWL, GA I/2, 374, 378.

127 GWL, GA I/2, 261, 267 y 282.

128 GWL, GA I/2, 282.

129 Paimann, R., Die Logik und das Absolute. Fichtes Wissenschaftslehre zwischen Wort, Begriff und Unbegrieflichkeit (Königshausen \& Neumann, Würzburg, 2006) 48.

130 Principalmente: GWL, GA I/2, 261, 293 y 296-7.

131 GWL, GA I/2, 271.

132 Jürgensen, S., op cit., pp. 54 y ss. y Neuhouser, F., op. cit., p. 46.
} 
as de realidad y negación son en principio relativas (mediadas por la cantidad), la realidad del poner absoluto es "realidad sin más", es decir, realidad sin negación 133 y la negación que mienta el No-Yo absoluto no es sino negación absoluta (desaparición total) del Yo. Ingresada en el sistema, este tipo de negación refiere sólo a la condición de posibilidad de toda negatividad en general y por ende de toda representación sin más de un objeto. 134

En cuarto y último lugar, el vínculo entre la acción originaria de poner y la categoría de realidad por medio del principio de identidad no es exclusivo de esta categoría, como pareciera decir Fichte. 135 Pues él mismo sostiene que, al igual que todo elemento del sistema, toda categoría es deducida del Yo en cuanto sujeto absoluto. ${ }^{136}$

Más allá de lo que pueda afirmarse a partir de pasajes tomados en forma aislada de la GWL, Fichte no postula en ningún momento la primacía de la categoría de la realidad. Sin embargo, debe reconocerse que si tales lecturas son posibles, se debe a cierta falta de claridad en la GWL, algo que Fichte, por cierto, ya reconoce en el prefacio a esta obra. 137

\section{Consideraciones finales}

El presente trabajo permite apreciar en qué medida la reformulación de la deducción kantiana de las categorías constituye una de las líneas de investigación centrales dentro del proyecto fichteano, entre 1793 y 1795, de una sistematización, en sentido de Reinhold, de la filosofía de Kant. El proyecto de sistematizar el conjunto categorial, como se ha mostrado, ya aparece en manuscritos de Fichte entre 1793 y 1794, y, en este sentido, la deducción de las categorías de la GWL puede ser vista como el resultado de un camino que se inicia en las anotaciones de Fichte de fines de 1793 citadas al inicio de (2). La deducción que ofrece la GWL se desarrolla de acuerdo a los dos motivos identificados en las Meditaciones personales y en las lecciones de Zúrich: por un lado, la elaboración de una deducción que muestre el origen de las categorías como acciones originarias del Yo y, por ende, como generadas a partir del mismo en cuanto sujeto absoluto, y, por otro, el reordenamiento de la tabla kantiana de las categorías.

Un manuscrito de 1806/07 muestra cuán importante es para Fichte la deducción de las categorías dentro de la exposición misma de la GWL. Allí sostiene que los

\footnotetext{
133 GWL, GA I/2, 288.

134 GWL, GA I/2, 267.

135 GWL, GA I/261.

136 GWL, GA I/262.

137 GWL, GA I/2, 252 y ss. Véase además, en lo que concierne a la falta de claridad en los $\S \S 1-4$ de la GWL, las cartas a Reinhold del 28 de abril (GA III/2, 315) y 2 de julio de 1795 (GA III/2, 343-347).
} 
dos puntos que componen "la esencia" de la GWL son la afirmación de la forma absoluta de la reflexión, "Ichform", en cuanto fundamento de todo saber y el "agotamiento analítico=sintético de esta forma a partir del punto medio de una efectuación recíproca de la sustancialidad absoluta con la causalidad absoluta". ${ }^{138} \mathrm{La}$ importancia de la deducción ofrecida en GWL puede, a su vez, ser rastreada en la doctrina de la ciencia nova methodo. Si bien Fichte en estas lecciones propone un nuevo tipo de exposición para su sistema, 139 lo cual, como se explicará más adelante, quita centralidad a la deducción de las categorías, reafirma aquello que la presente investigación ha mostrado como el resultado principal de la reelaboración del sistema categorial kantiano en la GWL: por un lado, que "hay tres categorías fundamentales[:] subst[ancialidad,] causalidad y efectuación recíproca", $140 \mathrm{y}$, por otro, que la determinación recíproca es la categoría principal o "la categoría de las categorías". ${ }^{141}$ A pesar de la importancia de la deducción de las categorías y del primado de la determinación recíproca en la GWL, esta cuestión, salvo contadas excepciones, 142 no ha sido explorada en la literatura que estudia esta obra.

$$
* * *
$$

El intento de Fichte en su GWL de mejorar la deducción kantiana de las categorías implica, como se ha visto, un cambio no sólo en el concepto de categoría, sino también en el de deducción. A las distintas diferencias que se han anunciado en la introducción y explicado en (2), se debe añadir aquí una que es de suma importancia para comprender no sólo el desarrollo del pensamiento fichteano a partir de la Doctrina de la ciencia nova methodo ${ }^{143}$ sino también el desarrollo de la filosofía alemana de Kant a Hegel, ${ }^{144}$ a saber: la ausencia en la deducción fichteana de la distinción kantiana entre deducción metafísica y transcendental. 145

Tal distinción no aparece ni en las Meditaciones personales ni en la GWL.146 En el caso de sus lecciones de Zúrich, cuando Fichte elabora su concepto de deduc-

138 GA II/10, 29, Bericht über den Begriff der Wissenschaftslehre und die bisherigen Schicksale derselben, $1806 / 07$.

139 GA IV/2, 17, Nachschrift Halle, y GA IV/3, 329, Nachschrift Krause.

140 GA IV/3, 485, Nachschrift Krause, véase también GA IV/2, 205, Nachschrift Halle.

141 GA IV/3, 497, Nachschrift Krause, véase también GA IV/2, 229 y 233, Nachschrift Halle.

142 Véase más arriba nota 124.

143 Serrano M., V., "La deducción del espacio y el tiempo en los parágrafos 10 a 12 de la Doctrina de la ciencia nova método del manuscrito de Krause", Endoxa, 30 (2012),p. 257.

144 Beiser, F., German Idealism. The Struggle against subjectivism: 1781-1801, pp. 164-166.

$145 \mathrm{KrV}$ B 159. Acerca de ambas deducciones y de los problemas internos de la argumentación kantiana véase Allison, H. El idealismo trascendental de Kant: una interpretación y defensa, pról. y trad. de D. M. Granja Castro, Barcelona: Anthropos/México: Universidad Autónoma Metropolitana Iztapalapa, 1992, pp. 191 y ss.

146 Fichte habla antes bien de deducción de las categorías (GA II/3, 41 y 59, Eigne Meditationen) o de deducción de las acciones sintéticas (en cuanto categorías) sin más. (GWL, GA I/2, 284) 
ción, se refiere al concepto kantiano de deducción sólo en el sentido de la deducción transcendental, es decir, como respuesta a la pregunta "quid juris". ${ }^{147} \mathrm{La}$ anotación de Fichte de fines de 1793 sobre la cuestión genética de las categorías surge, por el contrario, de un comentario a un pasaje de la deducción metafísica kantiana (KrV §10, B 104-105).

En el contexto de la presente investigación puede decirse lo siguiente al respecto. Primero, que, si bien la forma en que las categorías de la cualidad aparecen en los $\S \S 1-3$ de la GWL corresponde de algún modo con lo que Kant llama deducción metafísica, dado que allí estas categorías son obtenidas mediante abstracción de los juicios respectivos, ${ }^{148}$ este procedimiento contradice, como se ha indicado en (4) lo que Fichte entiende por deducción en sentido estricto. Segundo, que en la GWL Fichte parece reunir ambos modos de deducción en un mismo procedimiento, dado que su deducción de las categorías busca demostrar tanto la necesidad, orden sistemático y origen a priori de las categorías (objeto de la deducción metafísica) ${ }^{149}$ como también demostrar la posibilidad de las mismas en "cuanto conocimiento a priori de objetos de una intuición en general"150 (objeto de la deducción transcendental). Tercero, que el desarrollo del sistema mismo en la GWL impide una aplicación cabal de la distinción kantiana entre deducción metafísica y trascendental, dado que la deducción se da en un momento del despliegue del sistema en el que la mayoría de los elementos presupuestos en la versión kantiana de la deducción de las categorías aún no han sido deducidos. ${ }^{151}$

$* * *$

Ciertamente los cambios conceptuales y metodológicos en el intento fichteano de una reformulación del sistema kantiano de las categorías no permiten considerar su deducción como una continuación stricto sensu del proyecto kantiano. Sin embargo, como se ha mostrado hasta aquí, la deducción fichteana no se desvía de líneas centrales de la filosofía kantiana tales como el postulado de que toda realidad es para el Yo, la espontaneidad absoluta del sujeto, el carácter cuantitativo/intensivo de la realidad, la inmanencia absoluta como punto de vista transcendental y la determinación de la tercera categoría de cada grupo como síntesis de las dos restantes. Ahora bien, a la luz de la comprensión que Fichte mismo hace en esos años

\footnotetext{
147 GA IV/3, 36, Züricher Vorlesungen, Nachschrift Lavater.

148 GWL, GA I/2, 261, 267 y 282.

$149 \mathrm{KrV}$ B 159 , у B 102-109.

150 Ibídem.

151 Baste aquí con señalar que las facultades de conocimiento son deducidas en sentido estricto en la sección última del $\S 4$, Deducción de la representacion, y que espacio y tiempo suponen, según afirma Fichte, la demostración de la idealidad de los objetos (GA I/2, 335 n.), lo cual también sucede luego de la deducción de las categorías en sentido estricto en la GWL.
} 
de su relación con Kant, la reconstrucción aquí ofrecida brinda elementos para entender por qué Fichte se ve como un continuador o mejorador de Kant en el caso de las categorías. La deducción de la GWL confiere al conjunto categorial una uniformidad y consistencia sistemáticas de las que carecen las categorías kantianas y provee, así, a la filosofía transcendental de mejores armas contra los ataques, principalmente, de Schulze y Maimon.

Sin embargo, también es cierto que la propuesta fichteana paga muy caro estos logros: en la GWL las categorías, como puede verse, pierden principalmente capacidad de explicar tanto la experiencia real como las condiciones de posibilidad del conocimiento objetivo. El sistema gana ciertamente en consistencia, pero pierde la referencia a aquello que Kant y Fichte buscan explicar.

$* * *$

Esta relación inversamente proporcional entre sistematicidad y capacidad de explicar tanto la experiencia real como las condiciones a priori del conocimiento objetivo no es de verse, por cierto, en las lecciones sobre la doctrina de la ciencia nova methodo. Esto se debe principalmente al cambio ya mencionado en el modo de exposición (y de deducción) del sistema. En estas lecciones Fichte abandona la división entre filosofía teórica y práctica característica del modo de exposición de la GWL y presente en el proyecto original en el que se enmarcan las Meditaciones personales. ${ }^{152} \mathrm{La}$ anulación de esta división resulta en una mejor articulación entre las categorías y la multiplicidad de la experiencia posible, dado que la deducción de las categorías en estas lecciones combina conceptos provenientes de lo que en la GWL son el saber teórico y el saber práctico. ${ }^{153}$ Una tal combinación resultaría, por cierto, imposible dentro de las exigencias metodológicas de la GWL.

Ahora bien, con la anulación de la división entre una parte teórica y otra práctica también desaparece el lugar central que la deducción fichteana de las categorías posee entre 1793 y 1795. La doctrina de la ciencia nova methodo prescinde, como consecuencia del cambio metodológico, de un teorema del saber teórico que, como en la GWL, deba ser agotado en sus posibilidades para así demostrar la necesidad del tránsito hacia el saber práctico. ${ }^{154}$ En la doctrina de la ciencia nova methodo este tránsito no debe ser demostrado, pues desde un inicio lo práctico y lo teó-

\footnotetext{
152 Este manuscrito consiste en la primera parte del sistema que Fichte está elaborando entre noviembre de 1793 y febrero de 1794. El mismo se ocupa principalmente de exponer la filosofía teórica y, en cuanto tal, se complementa con el intitulado Filosofía práctica de 1794 (GA II/3, 181-266), como es de verse en la primera parte de este segundo manuscrito que lleva como título "Tránsito como resultado de la investigación teórica". (GA II/3, 181)

153 Véase como ejemplo el tratamiento de la categoría de causalidad en el que los conceptos de fin y de voluntad juegan un rol central. (GA IV/3, 478-485)

154 GWL, GA I/2, 275-276. Vease también GA II/3, 176-177, Eigne Meditationen.
} 
rico se hallan interactuando. La importancia de la deducción de las categorías en la GWL reside, como se ha indicado en (3.2), precisamente en venir a resolver las contradicciones internas del teorema del saber teórico. La determinación del conjunto categorial como un conjunto sistemáticamente cerrado permite además, como se ha indicado en (4), postular la existencia de una actividad independiente y de una experiencia originaria de limitación (Anstoss), elementos con los que Fichte lleva el saber teórico hasta la pregunta acerca de las condiciones de posibilidad de una tal limitación o choque en el Yo y así demuestra la insuficiencia del saber teórico,155 con la que se justifica la necesidad del tránsito hacia lo práctico.

El cambio metodológico de la doctrina de la ciencia nova methodo permite sin embargo delimitar un primer momento en la recepción crítica que hace Fichte de la filosofía kantiana e identificar, como se ha intentado aquí, la cuestión de la sistematicidad de la deducción y ordenamiento de las categorías como una de las líneas principales del desarrollo del programa de una doctrina de la ciencia entre 1793 y 1795.

Emiliano Acosta

Vrije Universiteit Brussel/Ghent University emiliano.acosta@ugent.be

155 GWL, GA I/2, 362. 